\title{
Modeling and Optimization Issues Concerning a Circular Piezoelectric Actuator Design*
}

\author{
Steven J. Coorpender \\ Department of Mathematics \\ University of New Orleans \\ New Orleans, LA 70115 \\ Robert Sims \\ Department of Mathematics \\ University of Alabama at Birmingham \\ Birmingham, AL 35205
}

\author{
Daniel Finkel \\ Department of Mathematics \\ North Carolina State Univ. \\ Raleigh, NC 27695 \\ Alexandra B. Smirnova
Department of Mathematics
Kansas State University
Manhattan, KS 66502
}

\author{
Jennifer Kyzar \\ Department of Mathematics \\ University of Tennessee \\ Knoxville, TN 37919 \\ Mohamed Tawhid \\ Department of Mathematics \\ Univ. of Maryland Baltimore County \\ Baltimore, MD 21250
}

\author{
Chad E. Bouton \\ Battelle Memorial Institute \\ 505 King Avenue \\ Columbus, OH 43201
}

\author{
Ralph C. Smith ${ }^{\dagger}$ \\ Center for Research in Scientific Computation \\ North Carolina State University \\ Raleigh, NC 27695
}

\begin{abstract}
An electromechanical model for a circular piezoelectric actuator is developed and analyzed. A critical challenge in certain applications employing piezoceramic actuators is to maximize the displacement provided by the actuator while minimizing it power consumption. This problem is addressed here by developing an electromechanical model which can be used to optimize the volume displacement to admittance ratio for various circular actuator designs. The model includes multiple layers with independent radii which can be varied to optimize performance. The piezoceramic, bonding, plating, and mounting materials can be varied to accommodate various design criteria. Similarly, the model incorporates various options for boundary conditions. A significant advantage of the model lies in the property that for a variety of material configurations, analytic solutions can be obtained. Numerical examples demonstrating the properties of the model will be presented.
\end{abstract}

\section{Introduction}

This paper focuses on modeling and design issues concerning a thin piezoceramic actuator bonded to an underlying substrate material. Specifically, we consider a circular piezoceramic patch bonded to a circular metallic plate of larger radius. The edges of the plate are assumed to be clamped. Unimorph actuators of this type are commonly employed in applications ranging from micropositioners to precision valves, and in all cases, the accurate characterization of the displacements in the underlying plate due to applied voltages to the patch are crucial to the performance of the device.

The characterization of patch inputs to underlying structures, including plates, beams and shells, has been investigated from several perspectives. In all cases, the active contribution of the patches is

${ }^{*}$ This problem was investigated by the first six authors under the direction of the last two authors during the Industrial Mathematics Modeling Workshop for Graduate Students held at North Carolina State University on July 27-August 4, 1998. Partial support for this workshop was provided by the NSF Grant DMS 9704919.

${ }^{\dagger}$ Corresponding author: rsmith@eos.ncsu.edu, (919) 515-7552 
incorporated through the strains produced by the elongation of dipole moments in response to an applied field. The models differ in the manner through which these input strains are coupled with the dynamics of the underlying structures. As detailed in [3], PDE models quantifying the structural dynamics can be derived by incorporating the generated strains in appropriate energy formulations for the structures. The success of this approach for obtaining a highly accurate model for a circular plate with surface-mounted piezoceramic patches is illustrated in [1] with the experimental implementation of a corresponding modelbased controller described in [2]. While this approach provides a highly accurate model, the numerical solution of the modeling PDE can inhibit certain design considerations. In this paper, we consider a simplified model which characterizes the displacements of a fixed piezoceramic plate with a surface-mounted piezoceramic actuator in terms of an ODE which can be solved analytically. This analytic solution can then be employed to design actuators which provide optimal performance with minimal power input.

We note that aspects of this approach are analogous to those employed by Kim and Jones [4] in their analysis of patch contributions to thin plates. The approaches differ, however, in the geometries, solution techniques, and resulting optimality relations.

In Section 2, we derive the model for the composite structure. The derivation is considered in two steps. In the first, general ODE for the central circular plate/patch composite and outer plate annulus are derived for unspecified boundary conditions. In the second step, appropriate boundary conditions are derived and used to determine the unspecified coefficients in the inner and outer components, and hence characterize the displacement at any point in the plate. Attributes of the model are illustrated in Section 3 through consideration of design criteria such as the determination of an optimal patch radius and thickness.

\section{$2 \quad$ Structural Model}

For the development which follows, we consider a thin circular plate of radius $R_{0}$ and thickness $h$ having clamped edges. A circular patch having radius $R_{p e}$ and thickness $h_{p e}$ is bonded to one side of the plate as depicted in Figure 1. To simplify the discussion, we assume that the bonding layer is negligible and direct the reader to [3] for details regarding the inclusion of the bonding layer. The Young's modulus, flexural rigidity and Poisson ratio for the plate are respectively denoted by $E, D$ and $\nu$ while the notation $E_{p e}, D_{p e}$ and $\nu_{p e}$ is used to denoted commensurate characteristics of the patch. The transverse displacement of the plate is denoted by $w$. Finally, we assume that the plate is subjected to a uniform pressure $q$.

The model is developed by quantifying separately the displacements of the circular region composed of both the piezoceramic patch and plate and the displacement of the outer annular plate region. These components are then combined through the choice of appropriate boundary conditions.
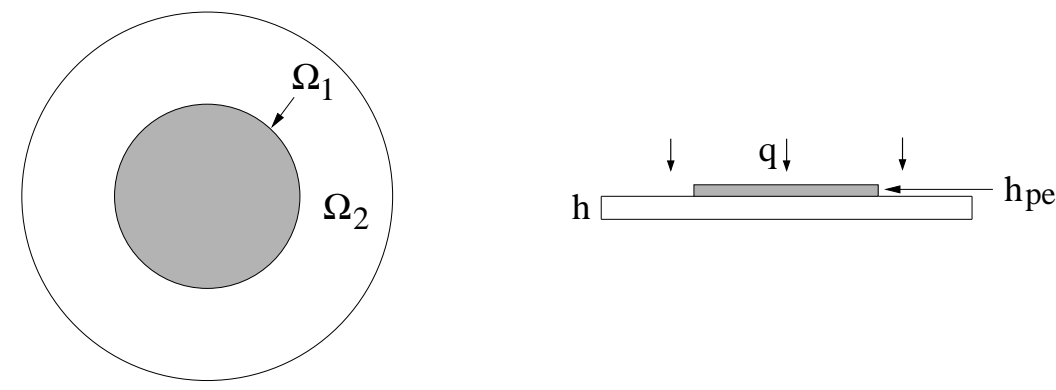

Figure 1. Thin circular plate with a surface mounted piezoceramic actuator. 


\subsection{Plate Model}

We first consider a general model for the annular region $\Omega_{2}$ of the structure which composed solely of the underlying plate. Under standard assumption for linear, thin, uniform plates, force and moment balancing yield the expression

$$
Q_{r}=\frac{1}{r} M_{r}+\frac{d M_{r}}{d r}-\frac{1}{r} M_{\theta}
$$

where $Q_{r}, M_{r}$ and $M_{\theta}$ respectively denote the radial shear resultant, radial moment result and tangential moment resultant $[3,5,6]$. Furthermore, it is illustrated in [5] that the moments can be expressed as

$$
\begin{aligned}
& M_{r}=D\left(\frac{d^{2} w}{d r^{2}}+\frac{\nu}{r} \frac{d w}{d r}\right) \\
& M_{\theta}=D\left(\nu \frac{d^{2} w}{d r^{2}}+\frac{1}{r} \frac{d w}{d r}\right) .
\end{aligned}
$$

Finally, the force resultant and pressure can be related through the expression

$$
2 \pi r Q_{r}=\pi r^{2} q
$$

To quantify the displacement due to the pressure, we combine (1)-(3) to obtain the differential equation

$$
\frac{d^{3} w}{d r^{3}}+\frac{1}{r} \frac{d^{2} w}{d r^{2}}-\frac{1}{r^{2}} \frac{d w}{d r}=\frac{r q}{2 D}
$$

or equivalently,

$$
\frac{d}{d r}\left[\frac{1}{r} \frac{d}{d r}\left(r \frac{d w}{d r}\right)\right]=\frac{r q}{2 D}
$$

The relation (4) can then be integrated to obtain

$$
w(r)=\frac{q r^{4}}{64 D}+\frac{c_{1} r^{2}}{4}+c_{2} \ln \left(\frac{r}{R_{0}}\right)+c_{3} \quad, \quad R_{p e} \leq r \leq R_{0}
$$

where $c_{1}, c_{2}$ and $c_{3}$ are constants which will ultimately be determined from the boundary conditions and interface conditions with the circular component $\Omega_{1}$.

The model for the composite material, comprised of the plate and piezoceramic patch and denoted by $\Omega_{1}$ in Figure 1, differs only in the expression of the flexural rigidity $D_{c}$. To specify $D_{c}$, it is necessary to first compute the height $z_{n s}$ of the neutral surface for the composite. This is accomplished by balancing forces and noting that the neutral surface denotes the locus of points which exhibit no strain under the action of a pure applied bending moment. If we let $\sigma_{r}(z)$ denote the radial stress at height $z$, then summation of forces over a differential element of the plate yields

$$
\int_{A} \sigma_{r} d A
$$

where $A$ is the cross-sectional area for the composite. Under the assumption that the plate is axisymmetric, and letting $\mathcal{R}$ denote the radius of curvature for the neutral surface, the formulation in terms of strains yields

$$
\int_{0}^{h} \frac{E}{\mathcal{R}}\left(z-z_{n s}\right) d z+\int_{h}^{h+h_{p e}} \frac{E_{p e}}{\mathcal{R}}\left(z-z_{n s}\right) d z .
$$


Integration then yields the expression

$$
z_{n s}=\frac{E_{p e} h_{p e}\left(h_{p e} / 2+h\right)+E h^{2} / 2}{E_{p e} h_{p e}+E h}
$$

for the neutral surface.

A homogenized flexural rigidity for the composite material is then given by

$$
D_{c}=\frac{1}{3}\left(\frac{E_{p e}}{1-\nu_{p e}^{2}}\right)\left[\left(h+h_{p e}-z_{n s}\right)^{3}-\left(h-z_{n s}\right)^{3}\right]+\frac{1}{3}\left(\frac{E}{1-\nu^{2}}\right)\left[\left(h-z_{n s}\right)^{3}-\left(-z_{n s}\right)^{3}\right] .
$$

It is noted that $D_{c}$ depends on both the material properties and dimensions of the component materials. It is also observed that for the region of the plate devoid of patch material $\left(h_{p e}=0\right.$ and $\left.z_{n s}=h / 2\right)$, the expression (6) reduces to the familiar relation

$$
D=\frac{E h^{3}}{12\left(1-\nu^{2}\right)}
$$

for a homogeneous plate.

The displacement $\widetilde{w}$ for the composite is then specified by the differential equation (4) with the flexural rigidity specified by (6). This then yields the general solution

$$
\widetilde{w}(r)=\frac{q r^{4}}{64 D_{c}}+\frac{c_{4} r^{2}}{4}+c_{5} \ln \left(\frac{r}{R_{0}}\right)+c_{6} \quad, \quad 0 \leq r \leq R_{p e}
$$

where the constants $c_{4}, c_{5}$ and $c_{6}$ are specified by the boundary and interface conditions.

\subsection{Boundary Conditions}

The relations (5) and (7) specify the displacements of the circular composite and annular plate region in terms of constants $c_{1}-c_{6}$ which are dependent upon the boundary conditions and interface conditions between the regions. To ensure compatibility between the two components and enforce mechanical boundary conditions, we enforce the following constraints.

To accommodate the clamp around the edge of the disk, we consider the fixed boundary conditions

$$
w\left(R_{0}\right)=w^{\prime}\left(R_{0}\right)=0 .
$$

A third constraint is provided by the implicit condition

$$
\widetilde{w}^{\prime}(0)=0
$$

which is used to enforce axial symmetry at the center of the disk. The remaining three constraints arise when enforcing compatibility between the composite disk and outer annular region. We first enforce equal displacements and slopes through the constraints

$$
\begin{aligned}
& w\left(R_{p e}\right)=\widetilde{w}\left(R_{p e}\right) \\
& w^{\prime}\left(R_{p e}\right)=\widetilde{w}^{\prime}\left(R_{p e}\right) .
\end{aligned}
$$

The third interface condition is the moment constraint

$$
\mathcal{M}_{r}\left(R_{p e}\right)=M_{r}\left(R_{p e}\right)
$$


which ensures that the radial moment in the annulus is equal and opposite when summed with the total moment for the composite. The former is defined in (2). The total moment for the composite is given by

$$
\mathcal{M}_{r}=M_{r}-M_{p e}
$$

where $M_{p e}$ denotes the moment generated by the piezoceramic patch when it strains in response to an applied voltage.

To specify $M_{p e}$, we first note that the free strains generated by the patch, for an input voltage $V$, are approximately linear and given by

$$
e_{p e}=\frac{V}{h_{p e}} d_{31} .
$$

The piezoelectric constant $d_{31}$ is typically estimated through a least squares fit to data, especially for patches which have aged or been repoled. The active moment generated by the patch is then

$$
\begin{aligned}
M_{p e} & =\int_{h}^{h+h_{p e}} \frac{E_{p e}}{1-\nu_{p e}} \cdot \frac{V}{h_{p e}}\left(z-z_{n s}\right) d z \\
& =\frac{E_{p e}}{2\left(1-\nu_{p e}\right)} V d_{31}\left(h_{p e}-2 z_{n s}+2 h\right) .
\end{aligned}
$$

The enforcement of the constraints (8)-(11) yields the expressions

$$
\begin{aligned}
& c_{1}=\frac{2}{1+\nu+(1-\nu) \frac{R_{0}^{2}}{R_{p e}^{2}}-\frac{D_{c}}{D}\left(1+\nu_{c}\right)\left(1-\frac{R_{0}^{2}}{R_{p e}^{2}}\right)}\left\{\frac{q R_{p e}^{2}}{16 D}\left[\nu_{c}-\nu+D_{c}\left(1+\nu_{c}\right)\left(\frac{1}{D}-\frac{1}{D_{c}}\right)\right]\right. \\
& \left.+\frac{q R_{0}^{4}}{16 D R_{p e}^{2}}\left[\nu-1-\frac{D_{c}}{D}\left(1+\nu_{c}\right)\right]-\frac{M_{p e}}{D}\right\} \\
& c_{2}=\frac{-q R_{0}^{4}}{16 D}-\frac{c_{1} R_{0}^{2}}{2} \\
& c_{3}=\frac{-q R_{0}^{4}}{64 D}-\frac{c_{1} R_{0}^{2}}{4} \\
& c_{4}=\frac{q R_{p e}^{2}}{8}\left(\frac{1}{D}-\frac{1}{D_{c}}\right)-\frac{q R_{0}^{4}}{8 R_{p e}^{2} D}+c_{1}\left(1-\frac{R_{0}^{2}}{R_{p e}^{2}}\right) \\
& c_{5}=0 \\
& c_{6}=\frac{-q R_{0}^{4}}{64 D}\left(\frac{1}{D}-\frac{1}{D_{c}}\right)+\frac{q R_{0}^{4}}{64 D}-\frac{q R_{0}^{4}}{16 D} \ln \left(\frac{R_{0}^{2}}{R_{p e}^{2}}\right)-\frac{c_{1} R_{0}^{2} \ln \left(R_{0}^{2} / R_{p e}^{2}\right)}{2}
\end{aligned}
$$

for the constants of integration. When employed in the displacement relations (5) and (7), this provides the capability for computing the displacement at any point which is due to the uniform pressure field and applied voltage to the patch. Representative results are provided in the next section. 


\section{Model Attributes}

The examples of this section illustrate various aspects about the model. The ultimate goal in these examples is the determination of parameters such the thickness of the underlying plate (mounting layer) $h$, the patch radius $R_{p e}$, and the patch thickness $h_{p e}$.

Example 1. Variation of Plate Thickness and Patch Radius

The radius of the plate was held constant at $4.0 \mathrm{~mm}$ and the thickness of the piezoelectric patch was held constant at 200 microns. We then varied the thickness of the plate and the radius of the piezoceramic patch in order to find the maximum deflection to voltage ratio as illustrated in Figures 2 and 3 . We observed the optimal thickness of the mounting layer to be 49.85 microns and the optimal radius of the piezoceramic layer to be $3.67 \mathrm{~mm}$.

\section{Example 2. Constant Plate Thickness}

Once the optimal thickness of the plate was determined, we investigated the effect of varying the radius of the piezoceramic patch. Figure 4 shows a cross section of deflection to voltage ratio versus radius of the piezoceramic patch with the constant plate thickness set at the optimal value of 49.85 microns. Note that the deflection drastically decreases when the radius extends beyond the optimal value of $3.67 \mathrm{~mm}$.



Figure 2. Relationship between plate thickness and patch radius. 


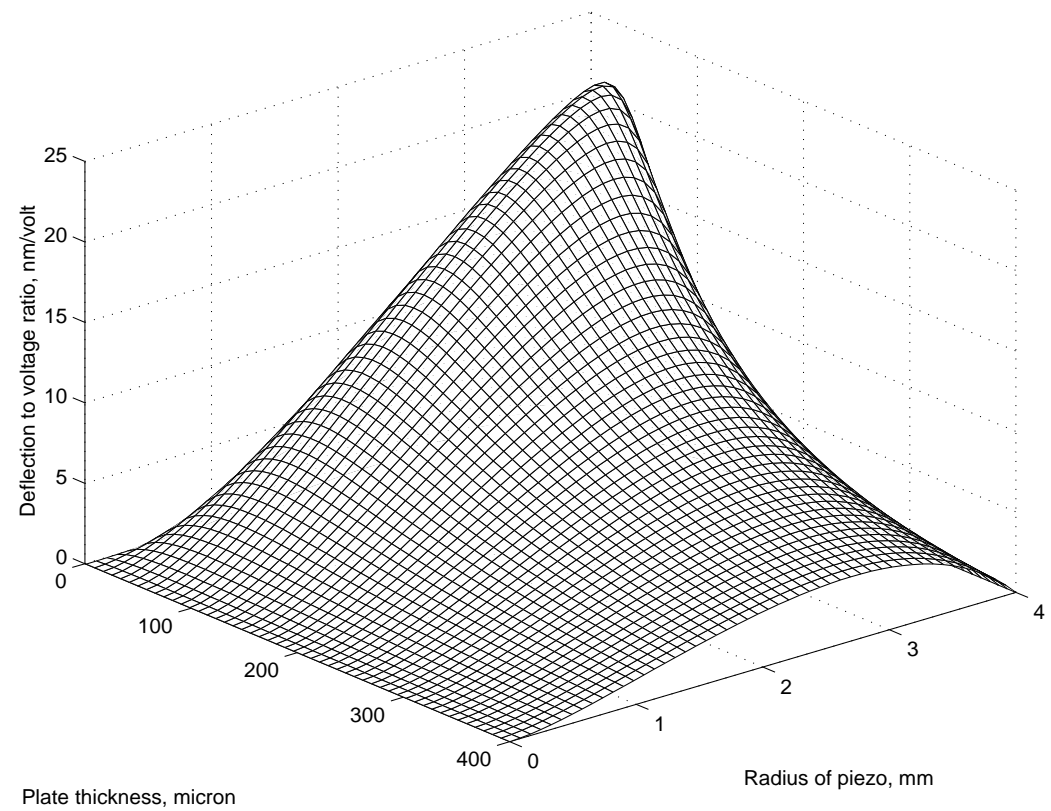

Figure 3. Relationship between plate thickness and patch radius.

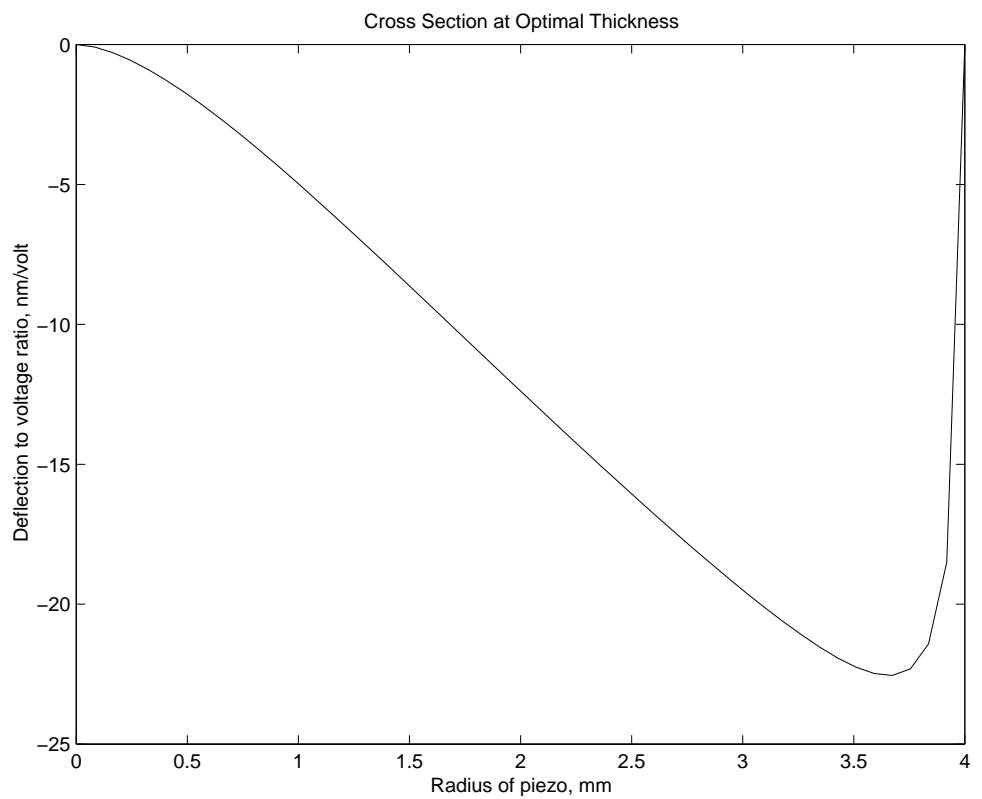

Figure 4. Deflection as a function of radius at the optimal patch thickness.

\section{Example 3. Constant Radius of Piezoceramic Patch}

Figure 5 illustrates a cross section of deflection to voltage ratio versus plate thickness with the radius of the piezoceramic held constant at its optimal value of $3.67 \mathrm{~mm}$. Note the optimal thickness is approximately 49 microns. The amount of deflection will decrease if the thickness is allowed to become too small.

We found the deflection for the optimal design to be $-22.54 \mathrm{~nm}$ as illustrated in Figure 6 . This is within the range of values observed by Battelle Memorial Institute in their applications. 


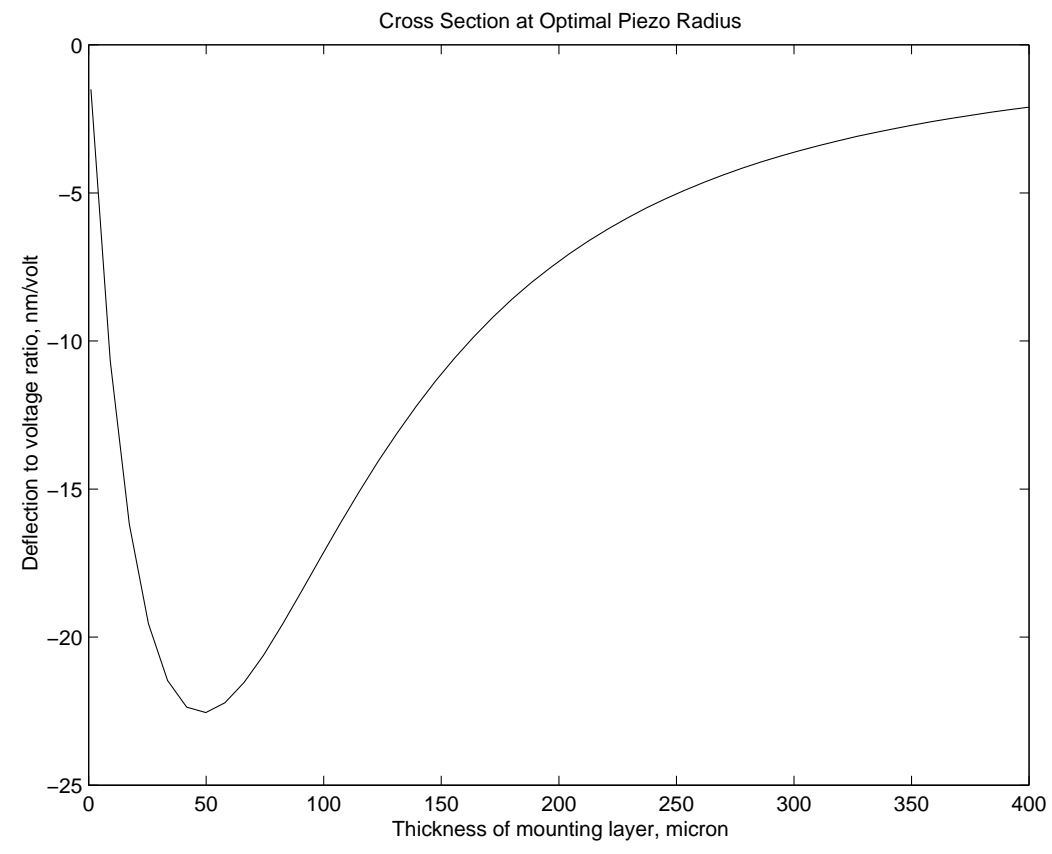

Figure 5. Deflection as a function of thickness at the optimal patch radius.

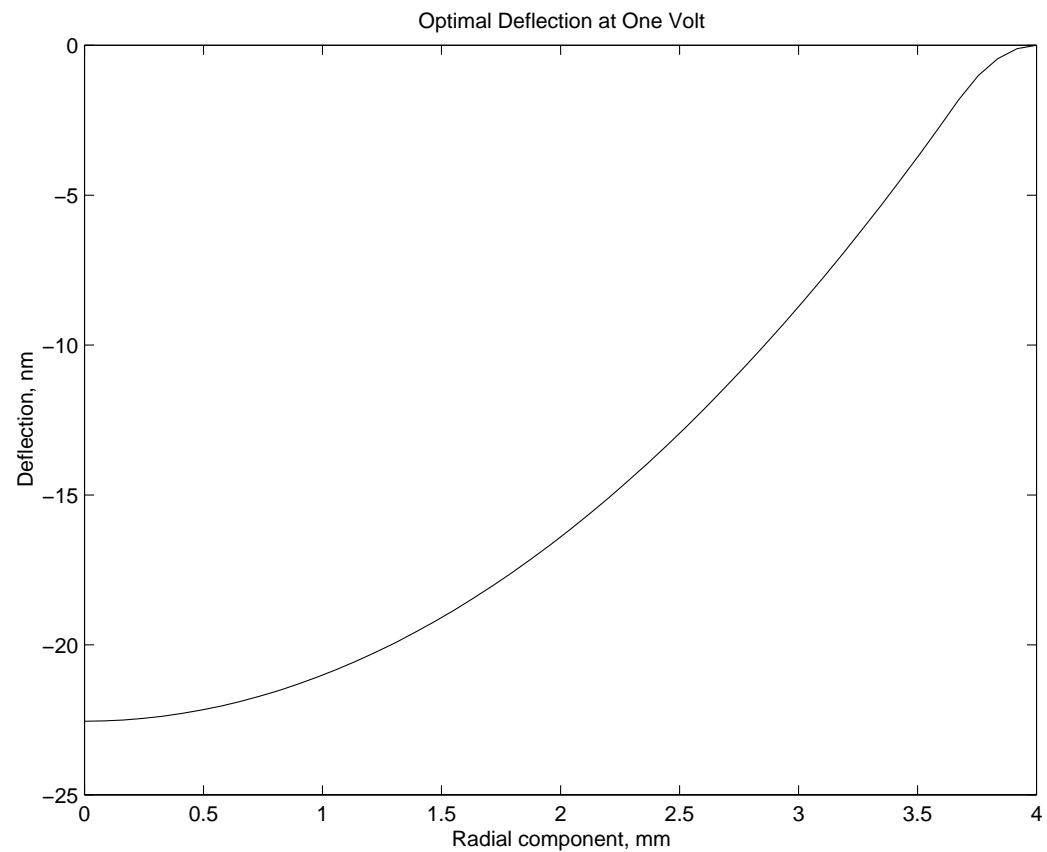

Figure 6. Optimal deflection at one volt 


\section{Concluding Remarks}

In this paper, we have considered the modeling of a circular plate with a surface-mounted circular piezoceramic patch in the center. By considering the structure to be composed of two regions which are radially and tangentially homogeneous (the inner composite comprised of the patch and substrate and the outer substrate annulus), analytic displacement relations were obtained. Constants of integration in the expressions were computed by enforcing boundary conditions for the plate along with compatibility relations between the two regions. We point out that the external moments generated by the patch in response to an applied voltage provide a component of the general moments which are balanced to provide one of the interface constraints.

The ease with which the model can be formulated and modified to accommodate changing material parameters or geometrical conditions makes it highly amenable to parametric studies or optimization investigations to determine optimal configurations. The effects of substrate thickness, patch radius and patch thickness on plate displacement for a fixed input voltage were illustrated in the examples.

\section{Acknowledgements}

We would like to thank Rich Fabiano, University of North Carolina, Greensboro, for his assistance during the workshop. We would also like to thank North Carolina State University and the Center for Research for Scientific Computation for providing us with the opportunity to attend the workshop.

\section{References}

[1] H.T. Banks, R.C. Smith, D.E. Brown, V.L. Metcalf and R.J. Silcox, "The estimation of material and patch parameters in a PDE-based circular plate model," Journal of Sound and Vibration, 199(5), 1997, pp. 777-799.

[2] H.T. Banks, R.C. Smith, D.E. Brown, R.J. Silcox and V.L. Metcalf, "Experimental confirmation of a PDE-based approach to design of feedback controls," SIAM Journal on Control and Optimization, 35(4), 1997, pp. 1263-1296.

[3] H.T. Banks, R.C. Smith and Y. Wang, Smart Material Structures: Modeling, Estimation and Control, Masson/John Wiley, Paris/Chichester, 1996.

[4] S.J. Kim and J.D. Jones, "Optimization of piezo-actuator/substructure coupling for active noise and vibration control," Proceedings of the Conference on Recent Advances in Active Control of Sound and Vibration, Eds., C.A. Rogers and C.R. Fuller, Blacksburg, VA, April 15-17, 1991, pp. 78-91.

[5] A.W. Leissa, Vibration of Plates, NASA SP-160, 1969, Reprinted by the Acoustical Society of America through the American Institute of Physics, 1993.

[6] S. Timoshenko and S. Woinowsky-Krieger, Theory of Plates and Shells, Second Edition, McGraw-Hill Book Company, Inc., New York, 1987. 